\title{
Mean Percentage of Returns for Stock Market Linked Savings Accounts
}

\author{
Ling Feng ${ }^{1}$, Zhigang Huang ${ }^{1}$, Xuerong $\mathrm{Mao}^{2}{ }^{*}$ \\ 1 School of Economics and Management, Fuzhou University, China \\ ${ }^{2}$ Department of Mathematics and Statistics, \\ University of Strathclyde, Glasgow G1 1XH, U.K.
}

\begin{abstract}
Stock market linked savings accounts have become more and more popular. The returns of these accounts are random so the returns, even the initial capital, are not guaranteed. They are very much different from the familiar fixed-term-fixed-rate savings accounts. The aim of this paper is to perform the stochastic and numerical analysis on the stock market linked savings accounts in order to establish the theory on the mean percentage of return (MPR). We will mainly perform the case studies on 5 typical plans linked to the UK Financial Times Stock Exchange (FTSE) 100 Index, but the theory developed is fully illustrated so that it can be applied to other plans by the reader.
\end{abstract}

Key words: Stock market linked savings accounts, stochastic differential equations, random payoff, the FTSE 100 index, Monte Carlo simulation, Euler-Maruyama scheme.

\section{Introduction}

Income needs seem to be a top priority at the moment - and with low savings rates and top UK equity income funds yielding less than $4 \%$, it is perhaps easy to understand why. With savings rates continuing at their record lows, some savers are turning to alternatives. It is in this spirit that many financial institutions are offering stock market linked savings plans to those looking to combine a high yield opportunity with some protection against a falling stock market. Here are some of them:

P1 Target Income Deposit Plan: This plan from Investec is designed to repay your initial deposit and to deliver a pre-defined income if the UK Financial Times Stock Exchange (FTSE) 100 Index (we will simply use the Index from now on) increases over the 3 year term: (a) $13 \%$ income if the Index is higher after 3 years. (b) If after 3 years the Index is equal to or lower than its starting level, you will receive back only your initial deposit.

P2 Stock Market Linked Savings Bound: This plan from Santander is designed to repay your initial deposit and to deliver a return over the 5 year term: (a) $40 \%$ income if the Index is increased by more than $40 \%$ in 5 years. (b) If after 5 years, the Index is increased by a percentage between $0.5 \%$ and $40 \%$, you will receive an income by the same percentage. (c) If after 5 years the Index is not increased by more than $0.5 \%$, you will receive a $0.5 \%$ income.

P3 Deposit Kick Out Plan: This plan from Gilliat is a 5 year plan. If the index is at or above its starting value at the end of year 3,4 or 5 , you will receive your original capital back plus $35 \%$ income. But if the Index is lower at the end of any of these 3 years, you will not receive any growth and you will only receive your capital back.

${ }^{*}$ Corresponding author. E-mail: x.mao@strath.ac.uk 
P4 The Investec FTSE 100 Target Income Deposit Plan: This is a six year structured deposit plan linked to the performance of the Index. The Index is measured at the start of the Plan, and then on a yearly basis thereafter. If the average closing level of the Index for the five business days up to and including the anniversary date is higher than $90 \%$ of the Initial Index Level a fixed income of $5 \%$ gross will be paid to you. If the anniversary Index level is equal to or below $90 \%$ of the Initial Index Level no income will be paid for that year. However should the Index meet the required level on any future anniversary, any previously missed income payments will be added back and paid out.

P5 Income Accumulator Plan: This plan from Morgan Stanley is a six year structured income investment plan linked to the performance of the Index. It offers up to $6.5 \%$ each year, with income being accrued for each week the Index closes between 4,500 and 9,000 points if it closes outside of this range, no income will be added for that week. Income payments will be calculated and paid at the end of each quarter, the total income payment being proportionate to the number of Weekly Observation Dates the Index is between the 4,500 to 9,000 range. For example for a quarter consisting of 13 weeks, should the Index finish between the range on 11 of 13 Weekly Observation Dates, the quarterly income payment would be $1.375 \%((0.065 / 4) \times 11 / 13)$. Also included is some protection against a falling market, since your initial capital is returned in full unless the value of the Index at the end of the term is below 4,250 points. If the Index is lower, your initial investment will be reduced by $1 \%$ for each $1 \%$ that the Final Index Level is below the level of the Index at the start of the plan so you could lose some or all of your original capital.

All these plans are linked to the performance of the Index. The incomes of these plans are random. They are very much different from the familiar fixed-term-fixed-rated savings accounts. The index has increased by $14.43 \%$ in year 2013 (see Figure 1 in the end of paper). On the other hand, leading three to six year fixed rates are currently offering between $2.6 \%$ and $3.15 \%$, so the plans above look very attractive.

However, the past performance of the Index is not a guide to its future performance. In particular, recalling the past 17-year performance (see Figure 2 in the end of paper), one might feel the Index may have reached its peak and it will decline in the future (Plans P4 and P5 aim to attract these savers). So the returns of these plans, even the initial capital, are not guaranteed.

The aim of this paper is to perform the stochastic and numerical analysis on the stock market linked savings accounts in order to establish the theory on the mean percentage of return (MPR). We will mainly perform the case studies on Plans P1-P5 listed above, but the theory developed is fully illustrated so that it can be applied to other plans by the reader.

Our stochastic and numerical analysis is dependent on the stochastic models for the Index. There are many stochastic models developed in the past 50 years, including the geometric Brownian motion, the CRR model, the mean reverting square root process (see e.g. [1, 3, 4, 5, $7,8,10,13,14])$. In this paper we will use some of these known models to establish our new theory. We have to emphasize that it is certainly not the aim of this paper to model the Index. Our aim is to compute the mean payoffs for the stock market linked savings accounts should the Index follows a given stochastic model.

To illustrate the use of our theory, we will only compare the stock market linked savings accounts with the bank fixed term savings accounts by assuming that the savers choose only one of these two. Leading three to six year fixed rates are currently offering between $2.6 \%$ and $3.15 \%$ annually. For illustration, we will assume the annual fixed rate is $2.875 \%$ (the average of these two) throughout this paper. By the common sense, the saver will not choose a stock market linked savings account if the corresponding MPR is not greater than the percentage of the income of a fixed term savings account. For example, Plan P1 is not attractive if its MPR is not greater than $8.875 \%\left(=(1+0.02875)^{3}-1\right)$. 


\section{MPR based on Geometric Brownian Motion}

In this section we assume that the Index, denoted by $x(t)$, follows the geometric Brownian motion (GBM). The GBM was first used by Samuelson [16] in financial modelling. It was later used by Merton [14] and Black and Scholes [1] to derive their Nobel Prize winning pricing formula. Looking at Figure 1 and assuming that the Index will perform in a similar pattern in the future as year 2013, one would feel that the GBM is appropriate.

The GBM is described by the linear stochastic differential equation (SDE)

$$
d x(t)=\mu x(t) d t+\sigma x(t) d B(t), \quad t \geq 0,
$$

where $\mu, \sigma$ are both positive constants, known as the growth rate and the volatility, respectively, and $B(t)$ is a standard Brownian motion defined on a complete probability space $\left(\Omega, \mathcal{F},\left\{\mathcal{F}_{t}\right\}_{t \geq 0}, \mathbb{P}\right)$ with the filtration $\left\{\mathcal{F}_{t}\right\}_{t \geq 0}$ satisfying the usual conditions (i.e. it is right continuous and increasing while $\mathcal{F}_{0}$ contains all $\mathbb{P}$-null sets). Let us highlight that the unit for the time $t$ is year. We let $t=0$ be the starting date of the underlying plan and let $x(0)=x_{0}>0$ be the starting level of the Index. It is well known (see e.g. $[4,11,12]$ ) that the SDE has its explicit solution

$$
x(t)=x_{0} \exp \left(\left(\mu-0.5 \sigma^{2}\right) t+\sigma B(t)\right), \quad t \geq 0
$$

The Index in 2013 is shown in Figure 1 and its daily closing levels are listed in the Appendix. In 2013, there are 253 business (banking) days and we let $x_{i}(1 \leq i \leq 253)$ denote these levels. Moreover, the closing level of the Index on 31 Dec 2012 is 5897.81 and we set it as the starting level for 2013, namely $x_{0}=5897.81$. Assume that during this year, the Index obeys the SDE model (2.1). Let $h=1 / 253$. Then, by $(2.2)$, we have

$$
x(i h)=x((i-1) h) \exp \left(\left(\mu-0.5 \sigma^{2}\right) h+\sigma[B(i h)-B((i-i) h)]\right), \quad 1 \leq i \leq 253 .
$$

This implies immediately that

$$
\log (x(i h) / x((i-1) h)) \sim N\left(\left(\mu-0.5 \sigma^{2}\right) h, \sigma^{2} h\right) .
$$

Set $\xi_{i}=\log \left(x_{i} / x_{i-1}\right)$. Then $\left\{\xi_{i}\right\}_{1 \leq i \leq 253}$ is a sample of the normal distribution $N\left(\left(\mu-0.5 \sigma^{2}\right) h, \sigma^{2} h\right)$. It is therefore well known that the sample mean

$$
\bar{\xi}=\frac{1}{253} \sum_{i=1}^{253} \xi_{i}
$$

and variance

$$
s_{\xi}^{2}=\frac{1}{252} \sum_{i=1}^{253}\left(\xi_{i}-\bar{\xi}\right)^{2}
$$

give good estimations for $\left(\mu-0.5 \sigma^{2}\right) h$ and $\sigma^{2} h$, respectively. In other words, the estimated growth rate $\hat{\mu}$ and volatility $\hat{\sigma}$ satisfy

$$
\left(\hat{\mu}-0.5 \hat{\sigma}^{2}\right) h=\bar{\xi} \quad \text { and } \quad \hat{\sigma}^{2} h=s_{\xi}^{2} .
$$

That is,

$$
\hat{\mu}=253\left(\bar{\xi}+0.5 s_{\xi}^{2}\right) \quad \text { and } \quad \hat{\sigma}^{2}=253 s_{\xi}^{2} .
$$

Using the data listed in the Appendix with $x_{0}=5897.81$, we easily get

$$
\hat{\mu}=0.1423 \text { and } \hat{\sigma}=0.1220 \text {. }
$$

A sample path of the solution to the $\operatorname{SDE}(2.1)$ with $\mu=0.1423$ and $\sigma=0.1220$ starting from $x_{0}=5897.81$ on $t \in[0,1]$ is shown in Figure 3 (in the end of paper).

In the remaining of this Section, we will often use these estimated parameters to illustrate our theory. We will also assume that all plans discussed in this paper start from 31 December 2013 and the starting level of the Index is 6749.09 (i.e. the closing level on 31 December 2013), unless otherwise specified. 


\subsection{Plan P1}

This is the simplest plan discussed in this paper. By (2.2), we have

$$
x(3)=x_{0} \exp \left(3\left(\mu-0.5 \sigma^{2}\right)+\sigma B(3)\right)=x_{0} \exp \left(3\left(\mu-0.5 \sigma^{2}\right)+\sqrt{3} \sigma Z\right),
$$

where $Z \sim N(0,1)$ as $B(3) \sim N(0,3)=\sqrt{3} Z$. (Please recall that the time is measured in years). It is easy to see that $x(3)>x_{0}$ if and only if

$$
Z>-\frac{\sqrt{3}\left(\mu-0.5 \sigma^{2}\right)}{\sigma}=: z_{1}
$$

where $=$ : means "denoted by". Hence

$$
\mathbb{P}\left(x(3)>x_{0}\right)=\mathbb{P}\left(Z>z_{1}\right)=1-N\left(z_{1}\right),
$$

where $N(x)$ is the cumulative probability function of the standard normal distribution, namely

$$
N(x)=\int_{-\infty}^{x} \frac{1}{\sqrt{2 \pi}} e^{-y^{2} / 2} d y, \quad x \in(-\infty, \infty)
$$

We recall that for Plan $\mathrm{P} 1$, the plan holder will receive a $13 \%$ return if $x(3)>x_{0}$ but nothing if $x(3) \leq x_{0}$. Hence the MPR for Plan P1 is

$$
\operatorname{MPR}=0.13 \times \mathbb{P}\left(x(3)>x_{0}\right)+0 \times \mathbb{P}\left(x(3) \leq x_{0}\right)=0.13\left(1-N\left(z_{1}\right)\right) .
$$

Recall that we assume that the annual fixed interest rate for 3-year fixed rate bounds is $2.875 \%$. By the common sense, one would not take Plan P1 if

$$
\mathrm{MPR} \leq(1+0.02875)^{3}-1=0.08875345 .
$$

If one figures out $\mu$ and $\sigma$ he can easily verify if (2.6) holds or not and then decide whether take Plan P1. For example, if one uses the parameters estimated by (2.4), namely $\mu=0.1423$ and $\sigma=0.1220$, then the MPR $=0.1263897$. In this case, he would take the plan.

On the other hand, observing that the Index has continuously grown for 5 years (see Figure 2 ), he may feel the Index will not grow so fast in the future though the volatility may remain the same as before. He would like to know the range for the value of $\mu$ for which he would not take Plan P1. Note that (2.6) is equivalent to $N\left(z_{1}\right) \geq 0.3172812$, or $z_{1} \geq-0.4753151$, namely

$$
\frac{\mu-0.5 \sigma^{2}}{\sigma} \leq 0.2744233 \text {. }
$$

This implies $\mu \leq \sigma(0.2744233+0.5 \sigma)=0.04092164$. In other words, when the volatility is around 0.1220 , he would not take Plan P1 if $\mu \leq 4.1 \%$.

Formula (2.7) can be used in another way. Assume that one feels the growth rate is likely to be about $5 \%$ in the next 3 years and he wishes to know the volatility range for which he would not take Plan P1. In this case, $(2.7)$ becomes $0.5 \sigma^{2}+0.2744233 \sigma-0.05 \geq 0$, which implies $\sigma \geq 0.1442749$. In other words, when $\mu=0.05$, he would not take Plan P1 if $\sigma \geq 0.1442749$.

\section{$2.2 \quad$ Plan P2}

This plan is slightly more complicated than Plan P1. By (2.2), the percentage of increase of the Index in 5 years is $\hat{Z}-1$ (of course decrease when this is negative), where

$$
\hat{Z}:=\exp \left(5\left(\mu-0.5 \sigma^{2}\right)+\sqrt{5} \sigma Z\right)
$$


where $Z$ appears as $B(5) \sim N(0,5)=\sqrt{5} Z$ (similarly in a couple of places later) and $:=$ means "denotes". Hence the MPR for Plan P2 is

$$
\operatorname{MPR}=0.4 \mathbb{P}(\hat{Z}-1>0.4)+\mathbb{E}\left((\hat{Z}-1) I_{\{0.05 \leq \hat{Z}-1 \leq 0.4\}}\right)+0.05 \mathbb{P}(\hat{Z}-1<0.05),
$$

where $I_{A}$ is the indicator function of set $A$, i.e. $I_{A}(x)=1$ if $x \in A$ and 0 otherwise. In a similar way as we did for Plan P1, we can show

$$
\mathbb{P}(\hat{Z}-1>0.4)=1-N\left(z_{2}\right), \quad \mathbb{P}(\hat{Z}-1<0.05)=N\left(z_{3}\right),
$$

where

$$
z_{2}=\frac{\log 1.4-5\left(\mu-0.5 \sigma^{2}\right)}{\sqrt{5} \sigma}, \quad z_{3}=\frac{\log 1.05-5\left(\mu-0.5 \sigma^{2}\right)}{\sqrt{5} \sigma} .
$$

Let us now compute

$$
\begin{aligned}
\mathbb{E}\left((\hat{Z}-1) I_{\{0.05 \leq \hat{Z}-1 \leq 0.4\}}\right) & =\mathbb{E}\left(\hat{Z} I_{\{1.05 \leq \hat{Z} \leq 1.4\}}\right)-\mathbb{P}(1.05 \leq \hat{Z} \leq 1.4) \\
& =\mathbb{E}\left(\hat{Z} I_{\{1.05 \leq \hat{Z} \leq 1.4\}}\right)-N\left(z_{2}\right)+N\left(z_{3}\right) .
\end{aligned}
$$

But

$$
\begin{aligned}
\mathbb{E}\left(\hat{Z} I_{\{1.05 \leq \hat{Z} \leq 1.4\}}\right) & =\int_{z_{3}}^{z_{2}} \frac{1}{2 \pi} \exp \left(5\left(\mu-0.5 \sigma^{2}\right)+\sqrt{5} \sigma z-0.5 z^{2}\right) d z \\
& =\int_{z_{3}}^{z_{2}} \frac{1}{2 \pi} \exp \left(5 \mu-0.5(z-\sqrt{5} \sigma)^{2}\right) d z \\
& =e^{5 \mu} \int_{z_{3}-\sqrt{5} \sigma}^{z_{2}-\sqrt{5} \sigma} \frac{1}{2 \pi} \exp \left(-0.5 y^{2}\right) d y \\
& =e^{5 \mu}\left[N\left(z_{2}-\sqrt{5} \sigma\right)-N\left(z_{3}-\sqrt{5} \sigma\right)\right]
\end{aligned}
$$

Hence

$$
\mathbb{E}\left((\hat{Z}-1) I_{\{0.05 \leq \hat{Z}-1 \leq 0.4\}}\right)=e^{5 \mu}\left[N\left(z_{2}-\sqrt{5} \sigma\right)-N\left(z_{3}-\sqrt{5} \sigma\right)\right]+N\left(z_{2}\right)+N\left(z_{3}\right) .
$$

Substituting (2.10) and (2.11) into (2.8), we get the following theorem:

Theorem 2.1 For Plan P2, we have

$$
M P R=0.4-1.4 N\left(z_{2}\right)+1.05 N\left(z_{3}\right)+e^{5 \mu}\left[N\left(z_{2}-\sqrt{5} \sigma\right)-N\left(z_{3}-\sqrt{5} \sigma\right)\right],
$$

where $z_{2}$ and $z_{3}$ are defined by (2.10).

In the common sense, one would not take Plan P2 if

$$
\mathrm{MPR} \leq(1+0.02875)^{5}-1=0.152257 .
$$

In this paper we will use the software $\mathrm{R}$ to perform numerical calculations or the Monte Carlo simulations. It is convenient to design the following R-function on $\mu$ and $\sigma$ to compute the MPR for Plan P2:

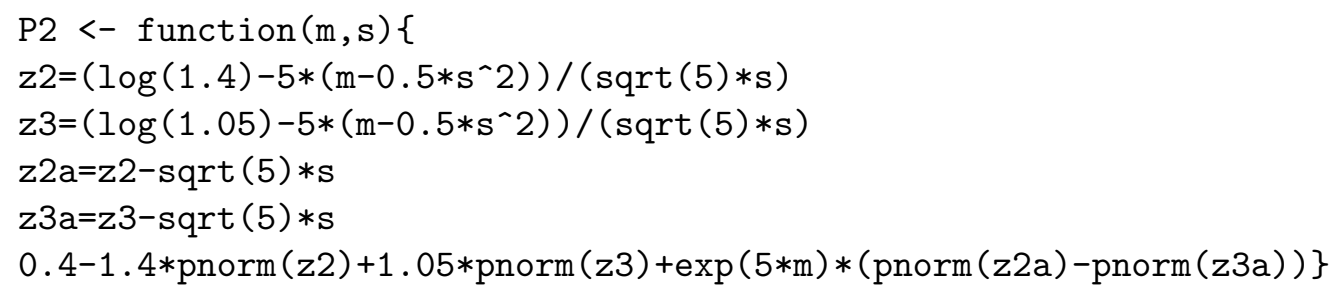


For example, if $\mu=0.1423$ and $\sigma=0.1220$ as estimated by (2.4), entering

$\mathrm{P} 2(0.1423,0.1220)$

in $\mathrm{R}$ we get the $\mathrm{MPR}=0.3832473$. In this case, one should take Plan P2. If we reduce the growth rate to $\mu=0.041$ but keep the volatility unchanged, then performing

$\mathrm{P} 2(0.041,0.1220)$

we get the MPR $=0.2094509$ so Plan P2 is still better than fixed term savings accounts. Recalling that one would not take Plan P1 when $\mu=0.041$ and $\sigma=0.1220$, we see Plan P2 seems offer a better return. If we further reduce the growth rate to $\mu=0.005$, the same as the basic rate set by the Bank of England, but still keep the volatility unchanged, then entering

$\mathrm{P} 2(0.005,0.1220)$

in $\mathrm{R}$ we get the MPR $=0.1301531$ so Plan $\mathrm{P} 2$ becomes not attractive.

\section{$2.3 \quad$ Plan P3}

This plan is more complicated than the two before. It is known that the solution of equation (2.1) is a homogeneous Markov process. Given any $x(s)=x>0$ at $s \geq 0$, equation (2.1) has the solution

$$
x(s+t)=x \exp \left(\left(\mu-0.5 \sigma^{2}\right) t+\sigma \sqrt{t} Z\right), \quad t \geq 0 .
$$

It is then easy to show

$$
\mathbb{P}(x(s+t) \leq y \mid x(s)=x)=N\left(z_{4}\right), \quad y>0,
$$

where

$$
z_{4}=\frac{\log (y / x)-\left(\mu-0.5 \sigma^{2}\right) t}{\sigma \sqrt{t}} .
$$

Hence the transition probability density of the solution is

$$
p(x ; y, t)=\frac{1}{\sqrt{2 \pi t} \sigma y} \exp \left(-\frac{\left[\log (y / x)-\left(\mu-0.5 \sigma^{2}\right) t\right]^{2}}{2 t \sigma^{2}}\right) .
$$

By the Markov property, we have

$$
\begin{aligned}
& \mathbb{P}\left(x(3) \geq x_{0}, x(4) \geq x_{0}, x(5) \geq x_{0}\right) \\
= & \mathbb{P}\left(x(3) \geq x_{0}\right) \mathbb{P}\left(x(4) \geq x_{0} \mid x(3) \geq x_{0}\right) \mathbb{P}\left(x(5) \geq x_{0} \mid x(4) \geq x_{0}\right) \\
= & \int_{x_{0}}^{\infty} p\left(x_{0} ; x_{3}, 3\right)\left(\int_{x_{0}}^{\infty} p\left(x_{3} ; x_{4}, 1\right)\left[\int_{x_{0}}^{\infty} p\left(x_{4} ; x_{5}, 1\right) d x_{5}\right] d x_{4}\right) d x_{3} .
\end{aligned}
$$

It is not difficult to show

$$
\int_{x_{0}}^{\infty} p\left(x_{4} ; x_{5}, 1\right) d x_{5}=1-N\left(d\left(x_{4}, 1\right)\right)
$$

where

$$
d(x, t):=\frac{\log \left(x_{0} / x\right)-\left(\mu-0.5 \sigma^{2}\right) t}{\sigma \sqrt{t}} \quad \text { for } x>0, t>0 .
$$

Hence

$$
\begin{aligned}
& \int_{x_{0}}^{\infty} p\left(x_{3} ; x_{4}, 1\right)\left[\int_{x_{0}}^{\infty} p\left(x_{4} ; x_{5}, 1\right) d x_{5}\right] d x_{4} \\
= & \int_{x_{0}}^{\infty} \frac{1}{\sqrt{2 \pi t} \sigma x_{4}} \exp \left(-\frac{\left[\log \left(x_{4} / x_{3}\right)-\left(\mu-0.5 \sigma^{2}\right)\right]^{2}}{2 \sigma^{2}}\right)\left[1-N\left(d\left(x_{4}, 1\right)\right)\right] d x_{4} .
\end{aligned}
$$


Setting

$$
\frac{\log \left(x_{4} / x_{3}\right)-\left(\mu-0.5 \sigma^{2}\right)}{\sigma}=: \bar{x}_{4}
$$

and noting

$$
d\left(x_{4}, 1\right)=\frac{\log \left(x_{0} / x_{3}\right)-\left[\sigma \bar{x}_{4}+2\left(\mu-0.5 \sigma^{2}\right)\right]}{\sigma}=d\left(x_{3}, 1\right)-\bar{x}_{4}-\left(\mu-0.5 \sigma^{2}\right) / \sigma,
$$

we get

$$
\begin{aligned}
& \int_{x_{0}}^{\infty} p\left(x_{3} ; x_{4}, 1\right)\left[\int_{x_{0}}^{\infty} p\left(x_{4} ; x_{5}, 1\right) d x_{5}\right] d x_{4} \\
= & \int_{d\left(x_{3}, 1\right)}^{\infty} \frac{1}{\sqrt{2 \pi}} e^{-0.5 \bar{x}_{4}^{2}}\left[1-N\left(\bar{x}_{4}\right)\right] d \bar{x}_{4} \\
= & \int_{d\left(x_{3}, 1\right)}^{\infty} \frac{1}{\sqrt{2 \pi}} e^{-0.5 \bar{x}_{4}^{2}}\left[1-N\left(d\left(x_{3}, 1\right)-\bar{x}_{4}-\left(\mu-0.5 \sigma^{2}\right) / \sigma\right)\right] d \bar{x}_{4} \\
= & 1-N\left(d\left(x_{3}, 1\right)\right)-\int_{d\left(x_{3}, 1\right)}^{\infty} \frac{1}{\sqrt{2 \pi}} e^{-0.5 \bar{x}_{4}^{2}} N\left(d\left(x_{3}, 1\right)-\bar{x}_{4}-\left(\mu-0.5 \sigma^{2}\right) / \sigma\right) d \bar{x}_{4} \\
= & 1-I\left(d\left(x_{3}, 1\right)\right),
\end{aligned}
$$

where

$$
I(x):=N(x)+\int_{x}^{\infty} \frac{1}{\sqrt{2 \pi}} e^{-0.5 \bar{x}_{4}^{2}} N\left(x-\bar{x}_{4}-\left(\mu-0.5 \sigma^{2}\right) / \sigma\right) d \bar{x}_{4}, \quad x \in \mathbb{R} .
$$

Substituting this into (2.21) we get

$$
\mathbb{P}\left(x(3) \geq x_{0}, x(4) \geq x_{0}, x(5) \geq x_{0}\right)=\int_{x_{0}}^{\infty} p\left(x_{0} ; x_{3}, 3\right)\left[1-I\left(d\left(x_{3}, 1\right)\right)\right] d x_{3} .
$$

In the same way as (2.19) was proved, we can then show

$$
\begin{aligned}
& \mathbb{P}\left(x(3) \geq x_{0}, x(4) \geq x_{0}, x(5) \geq x_{0}\right) \\
= & 1-N\left(d\left(x_{0}, 3\right)\right)-\int_{d\left(x_{0}, 3\right)}^{\infty} \frac{1}{\sqrt{2 \pi}} e^{-0.5 \bar{x}_{3}^{2}} I\left(-\sqrt{3} \bar{x}_{3}-4\left(\mu-0.5 \sigma^{2}\right) / \sigma\right) d \bar{x}_{3} .
\end{aligned}
$$

Recalling that the holder of Plan P3 will receive $35 \%$ income only if $x(3) \geq x_{0}, x(4) \geq x_{0}$ and $x(5) \geq x_{0}$ otherwise no income, we hence obtain the following theorem:

Theorem 2.2 For Plan P3, we have

$$
M P R=0.35\left(1-N\left(d\left(x_{0}, 3\right)\right)-\int_{d\left(x_{0}, 3\right)}^{\infty} \frac{1}{\sqrt{2 \pi}} e^{-0.5 \bar{x}_{3}^{2}} I\left(-\sqrt{3} \bar{x}_{3}-4\left(\mu-0.5 \sigma^{2}\right) / \sigma\right) d \bar{x}_{3}\right),
$$

where $d(x, t)$ and $I(x)$ are defined by (2.18) and (2.20), respectively.

Although the integration involved cannot be computed analytically, it can be done numerically. For illustration, let us assume that

$$
x_{0}=6500, \mu=0.025, \sigma=0.05 .
$$

So $d\left(x_{0}, 3\right)=-0.8227$ and

$$
\mathrm{MPR}=0.2781611-0.35 \int_{-0.8227}^{\infty} \frac{1}{\sqrt{2 \pi}} e^{-0.5 \bar{x}_{3}^{2}} I\left(-\sqrt{3} \bar{x}_{3}-1.9\right) d \bar{x}_{3} .
$$


Noting $0 \leq I(x) \leq 1$, the following formula is accurate enough

$$
\mathrm{MPR}=0.27813-0.35 \int_{-0.8227}^{4} \frac{1}{\sqrt{2 \pi}} e^{-0.5 \bar{x}_{3}^{2}} I\left(-\sqrt{3} \bar{x}_{3}-1.9\right) d \bar{x}_{3}
$$

(the error is less than $\left.0.35(1-N(4))=1.11 \times 10^{-5}\right)$. Also, noting

$$
I(x)=N(x)+\int_{-\infty}^{0} \frac{1}{\sqrt{2 \pi}} e^{-0.5 \overline{(x-u)^{2}}} N(u-0.475) d u, \quad x \in \mathbb{R},
$$

we see that

$$
\left|I(x)-N(x)-\int_{-3.525}^{0} \frac{1}{\sqrt{2 \pi}} e^{-0.5 \overline{(x-u)^{2}}} N(u-0.475) d u\right| \leq N(-4)=3.167124 \times 10^{-5} .
$$

It is therefore good enough to use

$$
I(x)=N(x)+\int_{-3.525}^{0} \frac{1}{\sqrt{2 \pi}} e^{-0.5(x-u)^{2}} N(u-0.475) d u, \quad x \in \mathbb{R} .
$$

Moreover, we have that $-\sqrt{3} \bar{x}_{3}-1.9=-0.4749$ and -8.8283 when $\bar{x}_{3}=-0.8227$ and 4 , respectively. We can now easily compute the MPR using (2.25) numerically. Inputting the following R-codes in $\mathrm{R}$

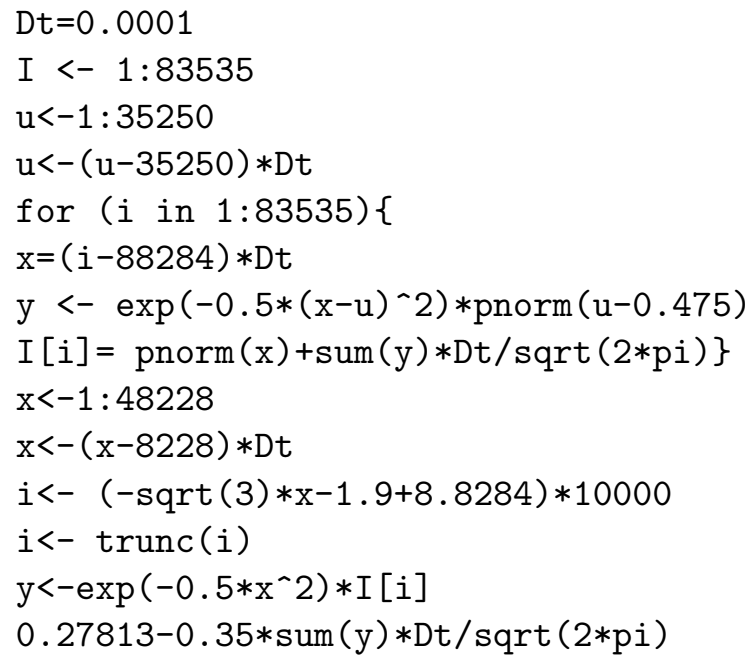

will give us the MPR $=0.2564508$. This is larger than $(1+0.02875)^{2}-1=0.152257$ so Plan P3 is more attractive than a 5-year fixed rate savings account. Let us point out that the numerical calculations above for the integrals involved use the Riemann sums with sufficiently small partitions $(\mathrm{Dt}=0.0001)$ so it is almost accurate.

Let us now introduce the Monte Carlo simulation to compute the MPR approximately. The linear $\operatorname{SDE}(2.1)$ has its explicit solutions at $t=3,4,5$ :

$$
\begin{aligned}
& x(3)=x_{0} \exp \left(3\left(\mu-0.5 \sigma^{2}\right)+\sigma B(3)\right), \\
& x(4)=x(3) \exp \left(\left(\mu-0.5 \sigma^{2}\right)+\sigma(B(4)-B(3))\right), \\
& x(5)=x(4) \exp \left(\left(\mu-0.5 \sigma^{2}\right)+\sigma(B(5)-B(4))\right) .
\end{aligned}
$$

Making use of these solutions, we can simulate a sample of large size for $(x(3), x(4), x(5))$. By the large number theory, the proportion of $\min (x(3), x(4), x(5)) \geq 6500$ gives a good estimation for the probability $\mathbb{P}(\min (x(3), x(4), x(5)) \geq 6500)$, and hence we will be able to obtain the MPR approximately. For this purpose, let us design an R-function: 


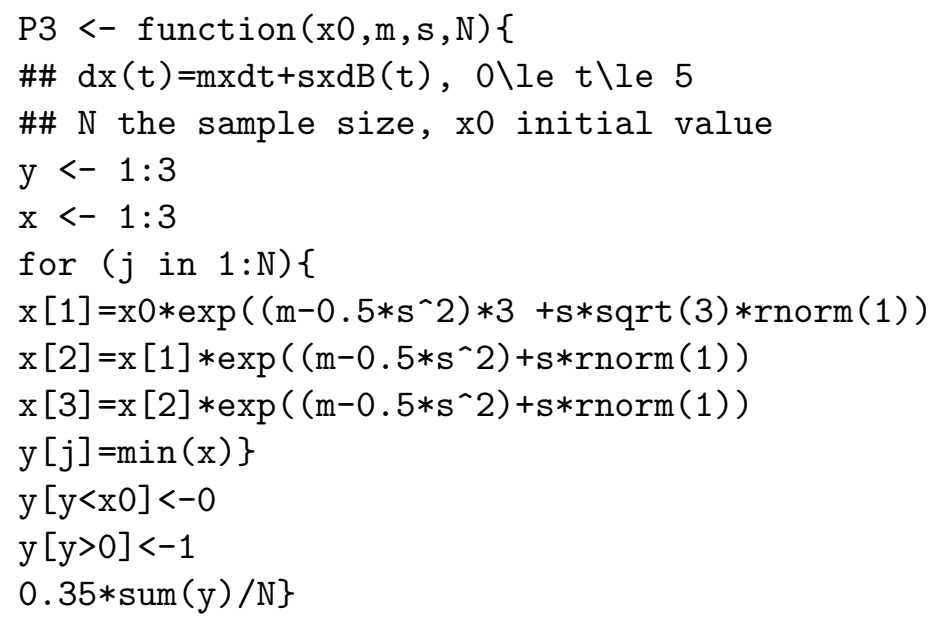

Now, we enter

$>$ P3 $(6500,0.025,0.05,50000)$

in $\mathrm{R}$ and get the MPR 0.256816 , which is very close to 0.2564508 obtained above. Of course, every time the function P3 is performed, a slightly different value for the MPR is produced. This is one difference between the Monte Carlo simulations and the numerical calculations above. However, the value should be around 0.2564 as long as the sample size $N$ is sufficiently large (we use $N=50000$ ). It is a good idea to perform P3 a number of times and then take the average of the values as the MPR. For example, performing P3 ten times

MPR $<-1: 10$

for $(j$ in $1: 10)\{$

$\operatorname{MPR}[j]<-P 3(6500,0.025,0.05,50000)\}$

$\operatorname{sum}(M P R) / 10$

we get the average value 0.2563561 for the MPR, which is more accurate. This example has shown the accuracy of the Monte Carlo simulations. There are other advantages. For example, it is simple than the numerical calculations above. The computation time is also much less than the numerical calculations (the reader may try and see the difference).

Moreover, the R-function P3 can handle different SDE parameters and initial values easily. For example, assume that Plan P3 starts from 31 December 2013 and the starting level of the Index $x_{0}=6749.09$, the closing level of the Index on 31 December 2013. Moreover, we use the growth rate and volatility as estimated by (2.4), namely $\mu=0.1423$ and $\sigma=0.1220$. Performing

P3 $(6749.09,0.1423,0.1220,50000)$

we get the $\mathrm{MPR}=0.337736$. Keeping $\sigma$ unchanged but reducing $\mu$ to $0.041,0.02$, and further to 0.005 , we get the MPR 0.206402, 0.157339, and 0.122871, respectively. These Monte Carlo simulations show Plan P3 is more attractive than 5-year fixed rate savings accounts if $\mu \geq 2 \%$ and the volatility is around 0.1220 .

The further benefits of the Monte Carlo simulations are demonstrated when we discuss the other plans in the following sections where the analytical solutions become too complicated.

\subsection{Plan P4}

This plan is even more complicated and we do not have an analytical solution yet but we will develop a Monte Carlo method to compute the MPR approximately. 
First of all, we observe that there are 253 business days per year (ignoring the leap year). We next assume that the plan holder will deposit his income, if any, at each anniversary to a fixed term bank savings account and the annual interest rate is $r=0.02785$ (as we assume in this paper). Moreover, the initial index level is the closing level of the Index at the start date of the plan. We may regard the initial index level as unit 1 (i.e. scaled by 6749.09 so $x_{0}=1$ ), as the SDE model (2.1) is linear and the annual income depends on the percentage change of the Index.

Let us now consider the closing level of the Index for the five business days up to and including the first anniversary date. Set $h=1 / 253$ and let $Z_{1}, \cdots, Z_{5}$ follow the standard normal distribution $N(0,1)$ independently. Then, these five closing levels are

$$
x(1-4 h)=\exp \left(\left(\mu-0.5 \sigma^{2}\right)(1-4 h)+\sigma \sqrt{1-4 h} Z_{5}\right)
$$

and

$$
x(1-j h)=x(1-(j+1) h) \exp \left(\left(\mu-0.5 \sigma^{2}\right) h+\sigma \sqrt{h} Z_{j+1}\right), \quad j=3,2,1,0 .
$$

The payment index level (PIL) for year 1 is

$$
\mathrm{PIL}_{1}=\frac{1}{5} \sum_{j=0}^{4} x(1-j h) .
$$

If $\mathrm{PIL}_{1}>0.9$, then $5 \%$ income is paid at the first anniversary date. The holder can then deposit it in a savings account and hence by the end of the Plan, he will get $0.05(1+r)^{5}$. If $\mathrm{PIL}_{1} \leq 0.9$, no payment is made at the first anniversary date. Similar, we can get $\mathrm{PIL}_{i}$ for year $i(2 \leq i \leq 6)$. Let $\mathrm{PIL}=\left(\mathrm{PIL}_{1}, \cdots, \mathrm{PIL}_{6}\right)$, i.e. the payment index level vector. For example, if $\mathrm{PIL}=(0.89,0.91,0.86,0.85,1.01,0.87)$, then at anniversaries $1,3,4$ and 6 , no payment is made, but at anniversaries 2 and $5,2 \times 5 \%$ and $3 \times 5 \%$ incomes are paid, respectively. In the end, the plan holder receives $2 \times 0.05(1+r)^{4}+3 \times 0.05(1+r)$ (percentage of) income.

Let us now design an R-function to perform the Monte Carlo simulations in order to get the MPR for this plan:

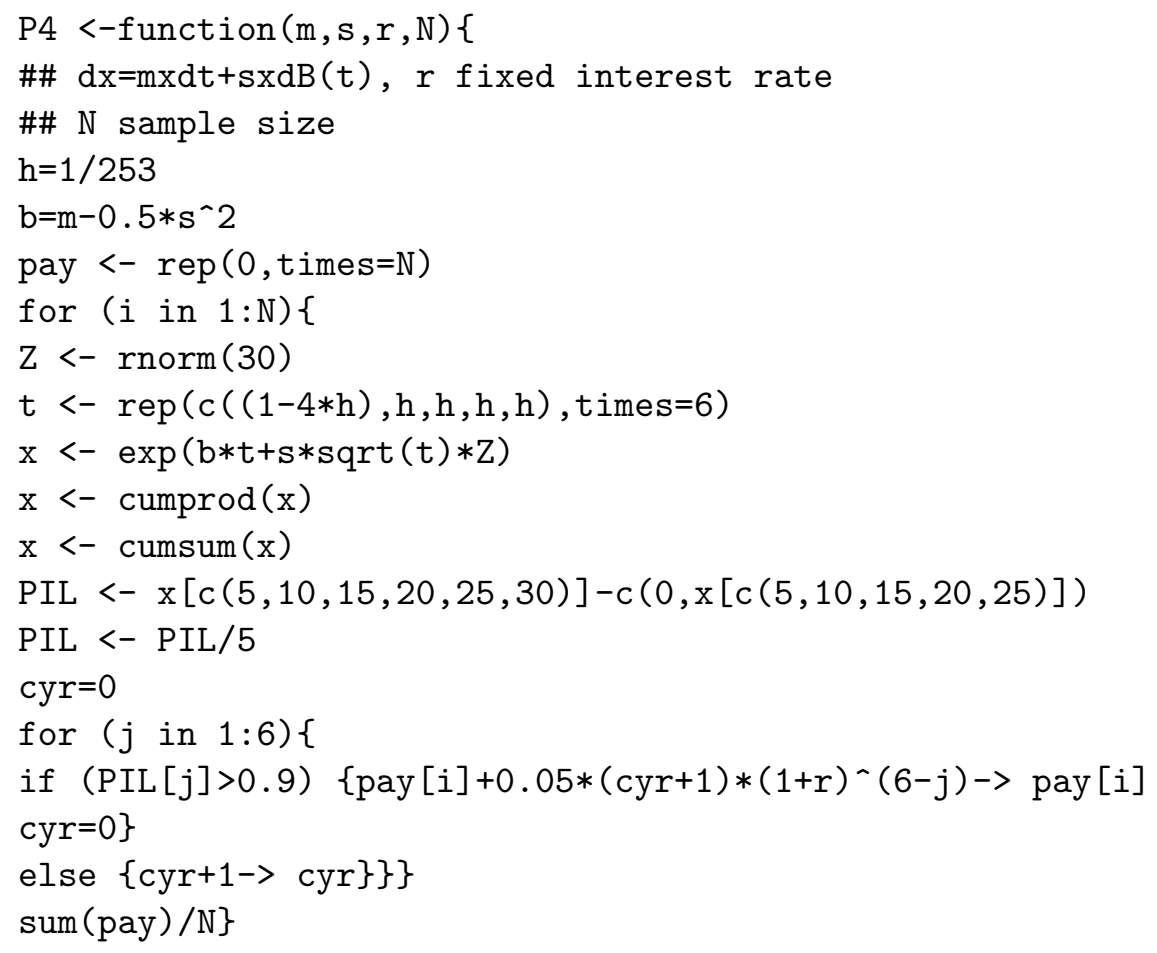

If we use the parameters estimated by (2.4), namely $\mu=0.1423$ and $\sigma=0.1220$, then 
shows the MPR $=0.3221625$. That is, the average percentage of return for 6 years is about $32.22 \%$ and this is certainly better than the 6 -year fixed term savings bound whose return is $18.54 \%$.

On the other hand, this plan is currently on offer in the UK market in order to attract the savers who have the feeling that the Index may have reached its peak and it will decline in the future (please recall Figure 2), as the plan even offers an income against a falling market up to $10 \%$. Let us assume $\mu=-0.02$ (the Index declines $2 \%$ per year in average) but leave the volatility the same as before. Then

$\mathrm{P} 4(-0.02,0.1220,0.02875,50000)$

shows the MPR $=0.2034199$ so Plan P4 is still better off. Further reduce $\mu$ to -0.03 , we get $\mathrm{MPR}=0.1828537$ so the plan almost makes even with the fixed-term savings accounts. To see the sensitivity of the volatility, we fix $\mu=-0.02$ but increase the volatility to $\sigma=0.15$. In this case, we have the MPR $=0.1963491$. These Monte Carlo simulations show that Plan P4 looks attractive unless the Index will decline more than $2 \%$ annually in the future.

\section{$2.5 \quad$ Plan P5}

This plan is most complicated one among the five plans we discuss in this paper. It has the features of Plans P2 and P4. There are 1520 business days in 6 years (taking leap years into account) so we may assume that there are 304 weeks and 5 business days every week. Assume also in years $1,2,4,5$, there are $12,13,13,13$ weeks in quarter $1,2,3,4$, respectively, but in years 3 and 6 , there are 12,13,12,13 weeks in quarter 1,2,3,4, respectively. Let $q w_{i}$ denote the number of weeks in the $i$ th quarter for these 24 quarters. For example, $q w_{1}=12$, qw $w_{2}=13$ etc. We also let $r_{w}$ denote the weekly fixed interest rate. So $\left(1+r_{w}\right)^{304}=1.02875^{6}$ which gives $r_{w}=0.0005595869$. Moreover, the annual income payment rate is $r_{p}=0.065$ so the quarterly income payment rate $r_{q p}=0.01625(0.065 / 4)$.

Set $h=6 / 304$ and let $Z_{1}, \cdots, Z_{304}$ follow $N(0,1)$ independently. Then the closing levels of the Index at the end of these 304 weeks are

$$
x_{j}=x(j-1) \exp \left(\left(\mu-0.5 \sigma^{2}\right) h+\sigma \sqrt{h} Z_{j}\right), \quad j=1,2, \cdots, 304,
$$

where $x(0)=x_{0}$. Define the payment indices

$$
\mathrm{PI}_{j}= \begin{cases}1 & \text { if } 4500 \leq x_{j} \leq 9000 \\ 0 & \text { otherwise }\end{cases}
$$

Then the proportion of the number of Weekly Observation Dates in quarter $i(1 \leq i \leq 24)$ when the Index is between the 4,500 to 9,000 range is

$$
p_{i}=\frac{1}{q w_{i}} \sum_{j=q_{i-1}+1}^{q_{i}} \mathrm{PI}_{j},
$$

where $q_{0}=0$ and $q_{i}=\sum_{k=1}^{i} q w_{k}$ (i.e. the total number of weeks from quarter 1 to $i$ ). So the income payment at the end of quarter $i$ is $r_{q p} p_{i}$, which can can be saved for $304-q_{i}$ weeks resulting the income $r_{q p} p_{i}\left(1+r_{w}\right)^{304-q_{i}}$ at the end of the plan. Thus, the total percentage of income over the 6 years is

$$
\sum_{i=1}^{24} r_{q p} p_{i}\left(1+r_{w}\right)^{304-q_{i}}
$$


and the mean percentage of income is

$$
\mathrm{MPI}=\mathbb{E}\left(\sum_{i=1}^{24} r_{q p} p_{i}\left(1+r_{w}\right)^{304-q_{i}}\right) .
$$

On the other hand, if the value of the Index at the end of the term, namely the Final Index Level (FIL), $x_{304}$ is below 4500 points, then initial investment will be reduced by $\left(x_{0}-x_{304}\right) / x_{0}$, which is the percentage of loss. Therefore, the mean percentage of loss in 6 years is

$$
\mathrm{MPL}=\mathbb{E}\left(I_{\left\{x_{304}<4500\right\}} \frac{x_{0}-x_{304}}{x_{0}}\right) .
$$

Consequently, the MPR of Plan P5 is

$$
\mathrm{MPR}=\mathrm{MPI}-\mathrm{MPL}
$$

The analysis above enables us to design an R-function compute the approximated MPR for this plan:

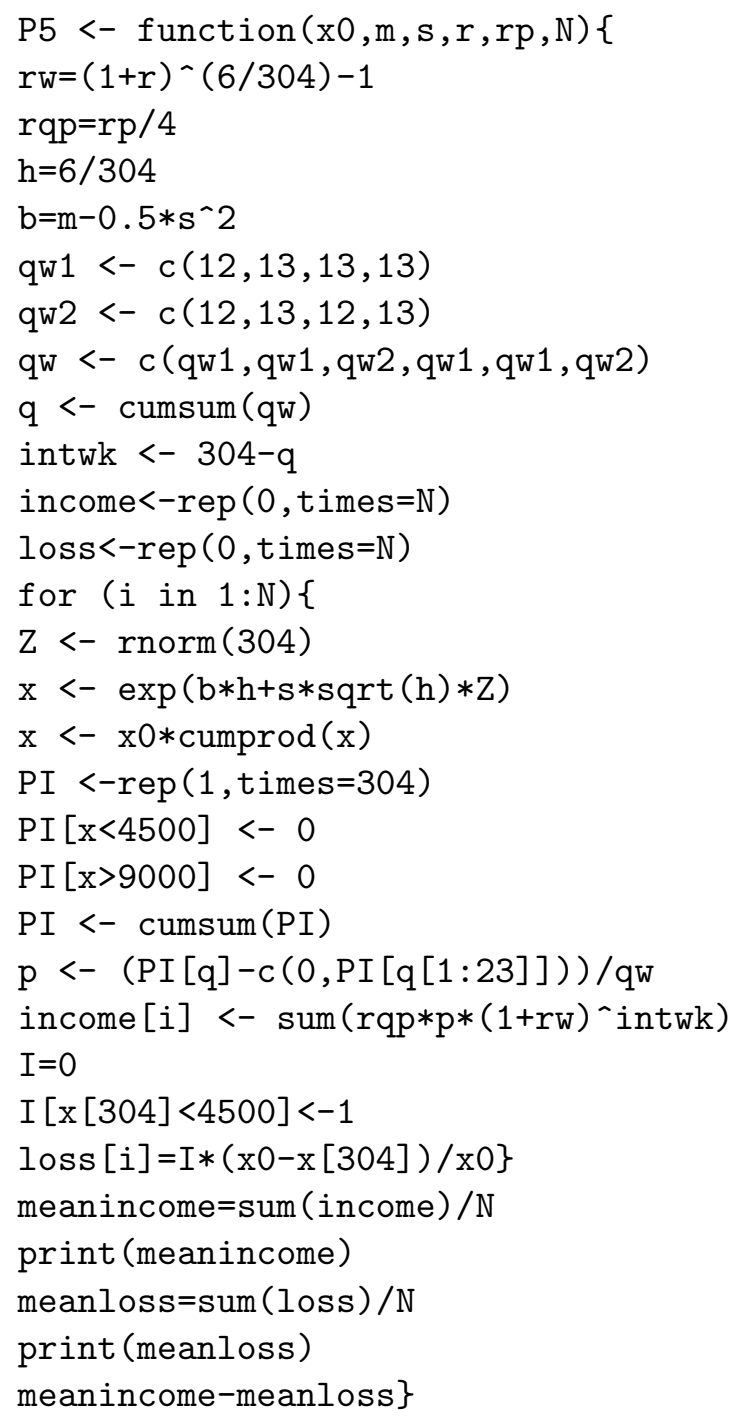

For example, if $x_{0}=6749.09, \mu=0.1423$ and $\sigma=0.1220$, entering 
in $\mathrm{R}$ we get the $\mathrm{MPI}=0.181587$ and $\mathrm{MPL}=7.344638 \times 10^{-6}$, and hence the $\mathrm{MPR}=0.1815797$. As the MPR is less than $\left.0.185384\left(=(1+r)^{6}-1\right)\right)$, Plan P5 is worse than the 6 -year fixed savings account in this case. This is because the growth rate 0.1423 is so large that the Index is very likely to exceed 9000 .

Looking at Figure 2, one may feel that the Index is likely to decline in the future. Assume $\mu=-0.03$ but leave $\sigma$ unchanged. Then entering

P5 $(6749.09,-0.03,0.1220,0.02875,0.065,50000)$

in $\mathrm{R}$ we get the $\mathrm{MPI}=0.3725448$ and $\mathrm{MPL}=0.1194941$, and hence the $\mathrm{MPR}=0.2530507$. In this case, Plan P5 is better than the 6-year fixed savings account.

There is a theoretical formula for the MPL, which will allow us to verify the Monte Carlo simulations. In fact, by the explicit formula (2.2), we have

$$
x_{304}=x_{0} \exp \left(6\left(\mu-0.5 \sigma^{2}\right)+\sqrt{6} \sigma Z\right),
$$

where $Z \sim N(0,1)$. It is easy to see that $x_{304}<4500$ if and only if

$$
Z<\frac{\log \left(4500 / x_{0}\right)-6\left(\mu-0.5 \sigma^{2}\right)}{\sqrt{6} \sigma}=: z_{5} .
$$

Hence

$$
\begin{aligned}
\mathrm{MRL} & =\int_{-\infty}^{z_{5}} \frac{1}{\sqrt{2 \pi}} e^{-0.5 z^{2}}\left[1-\exp \left(6\left(\mu-0.5 \sigma^{2}\right)+\sqrt{6} \sigma z\right)\right] d z \\
& =N\left(z_{5}\right)-\int_{-\infty}^{z_{5}} \frac{1}{\sqrt{2 \pi}} \exp \left(6\left(\mu-0.5 \sigma^{2}\right)+\sqrt{6} \sigma z-0.5 z^{2}\right) d z \\
& =N\left(z_{5}\right)-e^{6 \mu} \int_{-\infty}^{z_{5}} \frac{1}{\sqrt{2 \pi}} \exp \left(-0.5(z-\sqrt{6} \sigma)^{2}\right) d z \\
& =N\left(z_{5}\right)-e^{6 \mu} N\left(z_{5}-\sqrt{6} \sigma\right) .
\end{aligned}
$$

This can be computed in $\mathrm{R}$ easily. In fact, in the case when $x_{0}=6759.09, \mu=-0.03$ and $\sigma=0.1220$, entering

$\mathrm{x} 0=6749.09$

$\mathrm{m}=-0.03$

$\mathrm{s}=0.1220$

$\mathrm{z} 5=\left(\log (4500 / \mathrm{x} 0)-6 *\left(\mathrm{~m}-0.5 * \mathrm{~s}^{\wedge} 2\right)\right) /(\operatorname{sqrt}(6) * \mathrm{~s})$

pnorm $(z 5)-\exp (6 * m) * \operatorname{pnorm}(z 5-\operatorname{sqrt}(6) * s)$

we get the theoretical MPL $=0.119745$. We see the approximated MPL 0.1194941 obtained above by the Monte Carlo simulations is very close to this theoretical result.

The plan holder would hope the Index will not grow or decline too fast. Given the initial level is 6749.09 (almost right at the middle between 4500 and 9000), it is easy to see it is better for the growth rate to be around 0 . We can verify this by entering

P5 $(6749.09,0,0.1220,0.02875,0.065,50000)$

which yields the $\mathrm{MPR}=0.3322351$.

\section{MPR based on Mean Reverting SDE Models}

In the previous section, we have shown how to compute the MPR analytically or numerically or by the method of Monte Carlo simulations assuming the Index follows the GMB. On the other 
hand, if we look at the performance of the Index over the past 17 years (1997-2013) shown in Figure 2, we observe that the Index varies around 5200. It is therefore possibly more appropriate to use mean reverting SDEs models. There are several mean reverting SDEs models (see e.g. $[4,5,10,13])$. For example, the mean reverting process

$$
d x(t)=\mu(\lambda-x(t)) d t+\sigma x(t) d B(t)
$$

or the mean reverting square root process (also known as the CIR model [3])

$$
d x(t)=\mu(\lambda-x(t)) d t+\sigma \sqrt{x(t)} d B(t)
$$

or, more generally, the mean reverting theta process [10]

$$
d x(t)=\mu(\lambda-x(t)) d t+\sigma(x(t))^{\theta} d B(t)
$$

Due to the page limit, we will only use the CIR model (3.2) as an example to show how to perform Monte Carlo simulations to compute the MPRs for the stock market linked savings accounts. In this section, we will also assume the system parameters

$$
\mu=0.25, \quad \lambda=5200, \quad \sigma=10 .
$$

We should point out that it is not the aim of this paper to estimate these parameters based on the historical data but we refer the reader to e.g. $[2,15]$ in this direction. Figure 4 (in the end of paper) is a sample path of the CIR model (3.2) for 17 years with the parameters above and initial value 4000. This sample path is a Monte Carlo simulation based on the Euler-Maruyama (EM) scheme (see e.g. $[9,13])$ :

$$
x_{i}=x_{i-1}+\mu\left(\lambda-x_{i-1}\right) \Delta t+\sigma \sqrt{\left|x_{i-1}\right|} \Delta B_{i-1}, \quad 1 \leq i \leq T / \Delta t,
$$

where $\Delta t>0$ is the step size and $\Delta B_{i-1}=B(i \Delta t)-B((i-1) \Delta t)$. Higham and Mao [6] show that

$$
\lim _{\Delta t \rightarrow 0} \mathbb{E}\left(\sup _{1 \leq i \leq T / \Delta t}\left|x(i \Delta t)-x_{i}\right|^{2}\right)=0 .
$$

This strong convergence result forms the fundamental theory on the approximate MPRs by the Monte Carlo simulations developed in this section. Due to the page limit once again, we will not carry out the Monte Carlo approximations for all plans listed in Section 1 but only for Plans $\mathrm{P} 4$ and $\mathrm{P} 5$.

\subsection{Plan P4}

As in Section 2.4, we let $h=1 / 253$ and $r=0.02785$. As the SDE model (3.2) is nonlinear, we cannot scale the initial value to unit 1 but simply use what it is, namely $x_{0}=6749.09$. Define the payment index $(\mathrm{PI})$ for year $i(1 \leq i \leq 6)$

$$
\mathrm{PI}_{i}= \begin{cases}1 & \text { if } \frac{1}{5} \sum_{j=0}^{4} x(i-j h)>0.9 x_{0} \\ 0 & \text { otherwise }\end{cases}
$$

Set the payment index vector $\mathrm{PI}=\left(\mathrm{PI}_{1}, \cdots, \mathrm{PI}_{6}\right)$. Clearly, $\mathrm{PI}$ takes its values in the finite space $S:=\{0,1\}^{6}$. For example, if $\mathrm{PI}=(1,0,0,0,1,0)$, then at anniversaries $2,3,4$ and 6 , no payment is made, but at anniversaries 1 and $5,5 \%$ and $4 \times 5 \%$ incomes are paid, respectively. In the end, the plan holder receives $0.05(1+r)^{5}+4 \times 0.05(1+r)$ (percentage of) income. In this way, we can define uniquely an income function Pay : $S \rightarrow[0, \infty)$. Hence the MPR of Plan P4 is

$$
\operatorname{MPR}=\mathbb{E}(\operatorname{Pay}(\mathrm{PI}))
$$


We will use the EM scheme with step size $\Delta t$ to approximate $x(i-j h)$ for $i=1,2, \cdots, 6$ and $j=4,3,2,1,0$. Denote the EM approximation to $x(i-j h)$ by $X(i-j h)$. The approximate PI for year $i$ is then

$$
\mathrm{PI}_{i}(\Delta t)= \begin{cases}1 & \text { if } \frac{1}{5} \sum_{j=0}^{4} X(i-j h)>0.9 x_{0}, \\ 0 & \text { otherwise. }\end{cases}
$$

Let $\mathrm{PI}(\Delta t)=\left(\mathrm{PI}_{1}\left(\Delta t, \cdots, \mathrm{PI}_{6}(\Delta t)\right)\right.$.

Theorem 3.1 For Plan P4, we have

$$
M P R=\lim _{\Delta t \rightarrow 0} \mathbb{E}(\operatorname{Pay}(P I(\Delta t))) .
$$

Proof. By (3.6), we have

$$
\lim _{\Delta t \rightarrow 0} \mathbb{E}\left(\sup _{1 \leq i \leq 6,0 \leq j \leq 4}|x(i-j h)-X(i-j h)|^{2}\right)=0 .
$$

Define

$$
\bar{x}_{i}=\frac{1}{5} \sum_{j=0}^{4} x(i-j h) \quad \text { and } \quad \bar{X}_{i}=\frac{1}{5} \sum_{j=0}^{4} X(i-j h)
$$

for $1 \leq i \leq 6$. It then follows from (3.9) that

$$
\lim _{\Delta t \rightarrow 0} \mathbb{E}\left(\sup _{1 \leq i \leq 6}\left|\bar{x}_{i}-\bar{X}_{i}\right|^{2}\right)=0 .
$$

Recalling the definitions of $\mathrm{PI}_{i}$ and $\mathrm{PI}_{i}(\Delta t)$ as well as that the function Pay is defined on the finite space $S=\{0,1\}^{6}$, we see easily that the required assertion (3.8) holds if we can show

$$
\lim _{\Delta t \rightarrow 0}\left[\mathbb{P}\left(F_{i} \cap G_{i}^{c}\right)+\mathbb{P}\left(F_{i}^{c} \cap G_{i}\right)\right]=0, \quad 1 \leq i \leq 6,
$$

where $F_{i}=\left\{\bar{x}_{i}>0.9 x_{0}\right\}, F_{i}^{c}=\Omega-F_{i}$ and $G_{i}=\left\{\bar{X}_{i}>0.9 x_{0}\right\}, G_{i}^{c}=\Omega-G_{i}$. We now show this for $i=1$. Note that both $\bar{x}_{1}$ and $\bar{X}_{1}$ are continuously distributed random variables. Thus, for any $\epsilon \in(0,1)$, there is a sufficiently small $\delta>0$ such that

$$
\mathbb{P}\left(0.9 x_{0}<\bar{x}_{1}<0.9 x_{0}+\delta\right)+\mathbb{P}\left(0.9 x_{0}<\bar{X}_{1}<0.9 x_{0}+\delta\right)<\epsilon / 2 .
$$

Moreover, it follows from (3.13) that there is a sufficiently small $\Delta t^{*}>0$ such that

$$
\mathbb{P}\left(\left|\bar{x}_{1}-\bar{X}_{1}\right| \geq \delta\right)<\epsilon / 2, \quad \forall \Delta t<\Delta t^{*} .
$$

Therefore, for any $\Delta t<\Delta t^{*}$, we derive that

$$
\begin{aligned}
& \mathbb{P}\left(F_{i} \cap G_{i}^{c}\right)+\mathbb{P}\left(F_{i}^{c} \cap G_{i}\right) \\
\leq & \mathbb{P}\left(\left\{\bar{x}_{1} \geq 0.9 x_{0}+\delta\right\} \cap G_{i}^{c}\right)+\mathbb{P}\left(0.9 x_{0}<\bar{x}_{1}<0.9 x_{0}+\delta\right) \\
+ & \mathbb{P}\left(F_{i}^{c} \cap\left\{\bar{X}_{1} \geq 0.9 x_{0}+\delta\right\}\right)+\mathbb{P}\left(0.9<\bar{X}_{1}<0.9 x_{0}+\delta\right) \\
\leq & \mathbb{P}\left(\left|\bar{x}_{1}-\bar{X}_{1}\right| \geq \delta\right)+\mathbb{P}\left(0.9 x_{0}<\bar{x}_{1}<0.9 x_{0}+\delta\right) \\
+ & \mathbb{P}\left(0.9 X_{0}<\bar{X}_{1}<0.9 x_{0}+\delta\right) \\
< & \epsilon .
\end{aligned}
$$

This means that (3.11) holds for $i=1$. In the same way, we can show it holds for $i=2, \cdots, 6$. The proof is therefore complete.

The theorem shows that if we choose the step size $\Delta t$ sufficiently small, we then have $\mathbb{E}(\operatorname{Pay}(\operatorname{PI}(\Delta t))) \approx \mathrm{MPR}$. By the large number theory, if we simulate a sample of a large size for $\mathrm{PI}(\Delta t)$, its sample mean gives a good approximation for the MPR. The theory above leads us to design an R-function to perform the Monte Carlo simulations in order to get the MPR for this plan: 


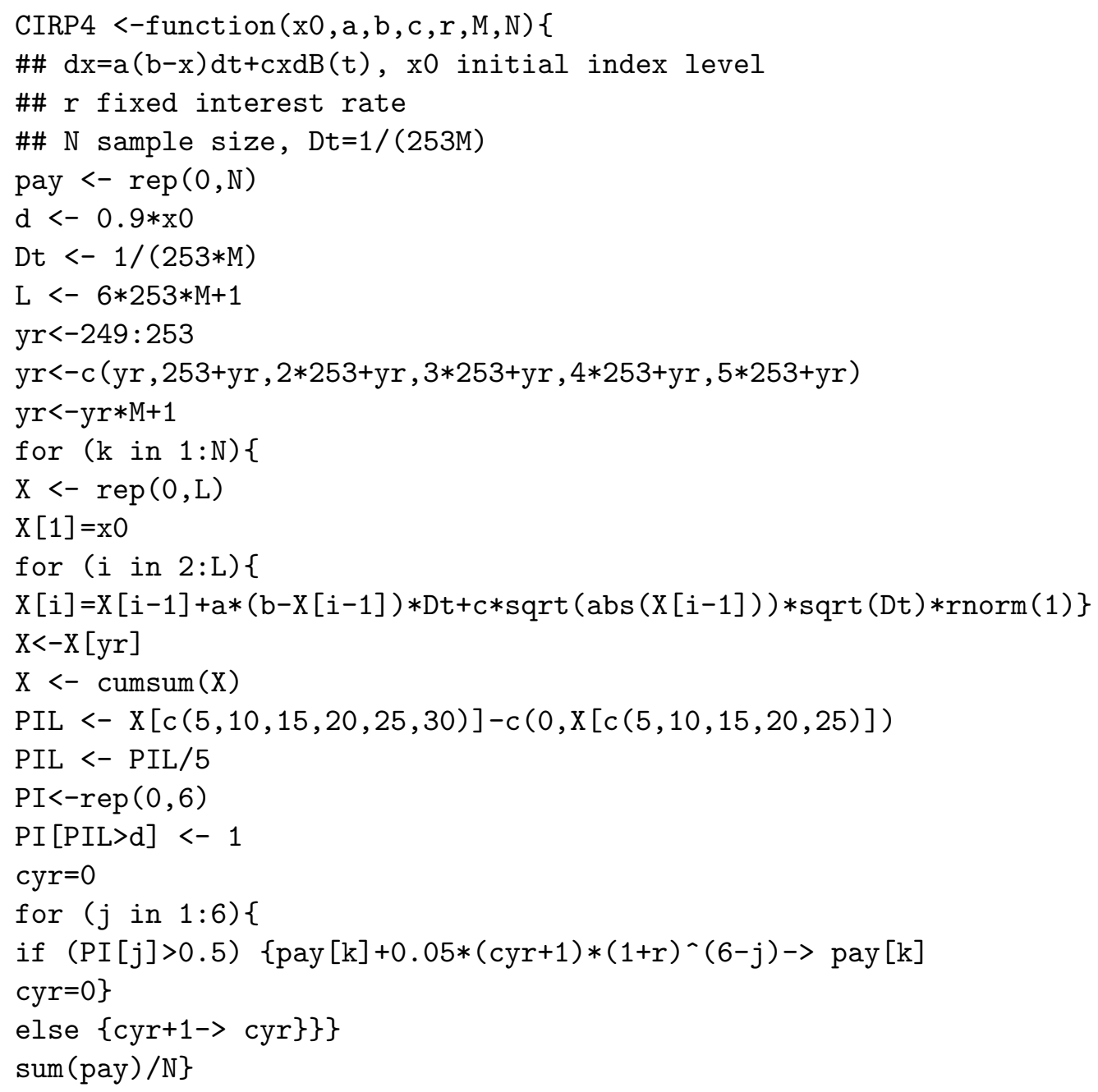

For example, using the parameters in (3.4), the step size $\Delta t=1 / 2530$ and the sample size 10000, by performing

CIRP4 $(6749.09,0.25,5200,10,0.02875,10,10000)$

we get the approximate $\mathrm{MPR}=0.1815733$. This indicates that Plan P4 is worse than a 6-year fixed rate bound, if the Index follows the CIR model (3.2) with the parameters specified in (3.4). This is not surprising as the mean index is 5200 and hence the index is very likely to be below $0.9 x_{0}=6074.18$.

\subsection{Plan P5}

We will use the same notation as in Section 2.5. Set $h=6 / 304$. Then the closing levels of the Index at the end of the 304 weeks are $x_{j}=x(j h)(1 \leq j \leq 304)$. Define the payment indices

$$
\mathrm{PI}_{j}= \begin{cases}1 & \text { if } 4500 \leq x_{j} \leq 9000 \\ 0 & \text { otherwise }\end{cases}
$$

and form the payment index vector $\mathrm{PI}=\left(\mathrm{PI}_{1}, \cdots, \mathrm{PI}_{304}\right)$. Clearly, $\mathrm{PI} \in S:=\{0,1\}^{304}$. Let us now define an income function Inc $: S \rightarrow[0, \infty)$ by

$$
\operatorname{Inc}(v)=\sum_{i=1}^{24} r_{q p} p_{i}\left(1+r_{w}\right)^{304-q_{i}}
$$


for $v=\left(v_{1}, \cdots, v_{304}\right) \in S$, where $q_{0}=0, q_{i}=\sum_{k=1}^{i} q w_{k}$ and

$$
p_{i}=\frac{1}{q w_{i}} \sum_{j=q_{i-1}+1}^{q_{i}} v_{j} \text {. }
$$

With this function, the income (percentage) over the 304 weeks is Inc(PI). On the other hand, if the value of the Index at the end of the term, namely the Final Index Level (FIL), $x_{304}$ is below 4500 points, then initial investment will be reduced by $\left(x_{0}-x_{304}\right) / x_{0}$, which is the percentage of loss. Therefore, the mean percentage of return in 6 year is

$$
\mathrm{MPR}=\mathbb{E}\left(\operatorname{Inc}(\mathrm{PI})-\frac{x_{0}-x_{304}}{x_{0}}\right)
$$

We will use the EM scheme with step size $\Delta t$ to get the approximate solutions $X_{j} \approx x_{j}$ for $1 \leq j \leq 304$. Define the approximate payment indices

$$
\mathrm{PI}_{j}(\Delta t)= \begin{cases}1 & \text { if } 4500 \leq X_{j} \leq 9000 \\ 0 & \text { otherwise }\end{cases}
$$

and form the approximate payment index vector $\mathrm{PI}(\Delta t)=\left(\mathrm{PI}_{1}(\Delta t), \cdots, \mathrm{PI}_{304}(\Delta t)\right)$.

Theorem 3.2 For Plan P5, we have

$$
M P R=\lim _{\Delta t \rightarrow 0} \mathbb{E}\left(\operatorname{Inc}\left(P I(\Delta t)-\frac{x_{0}-X_{304}}{x_{0}}\right) .\right.
$$

The proof is similar to that of Theorem 3.2 so is omitted. This theorem leads us to design the following R-function to perform the Monte Carlo simulations in order to get the approximate MPR for Plan P5:

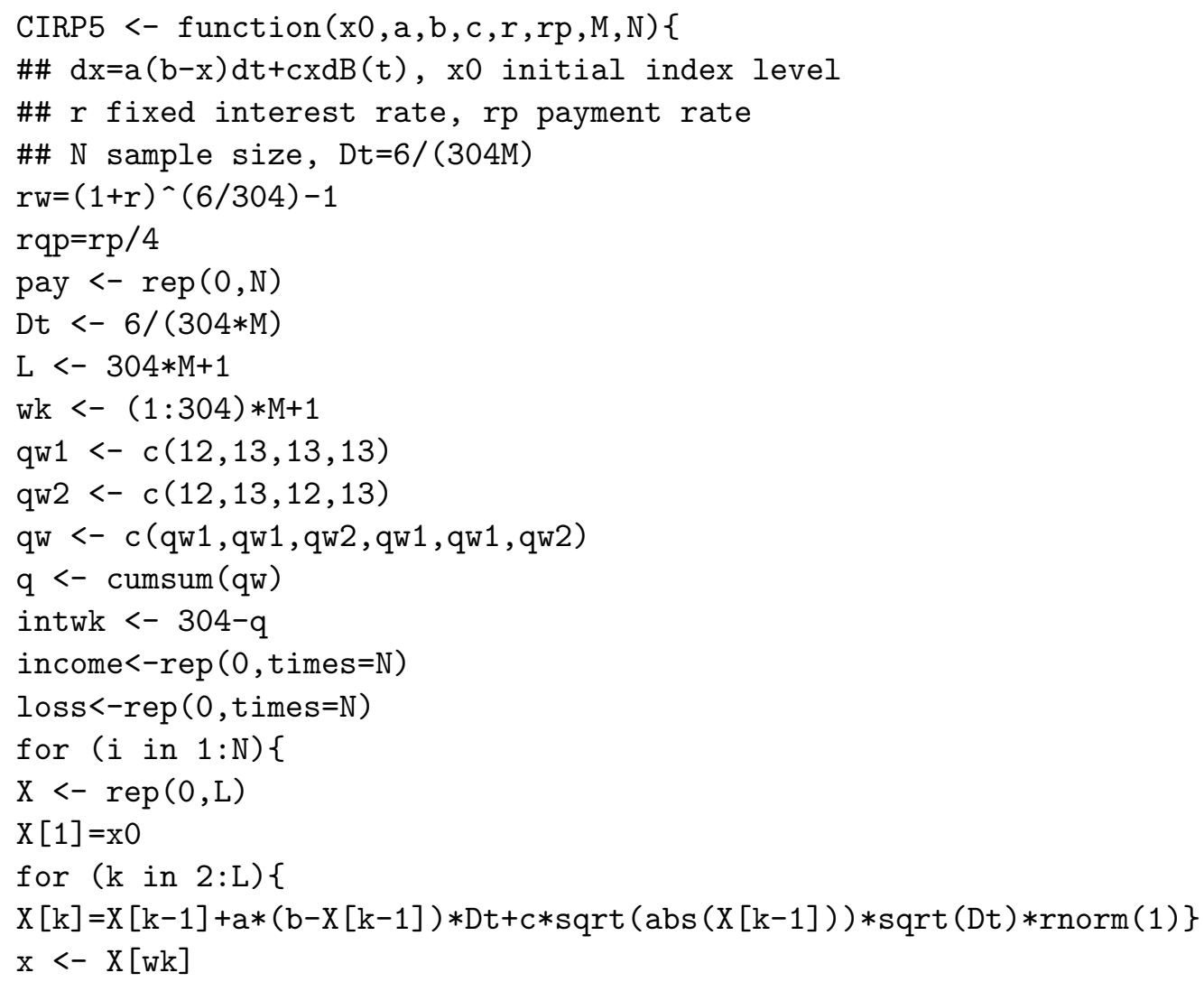




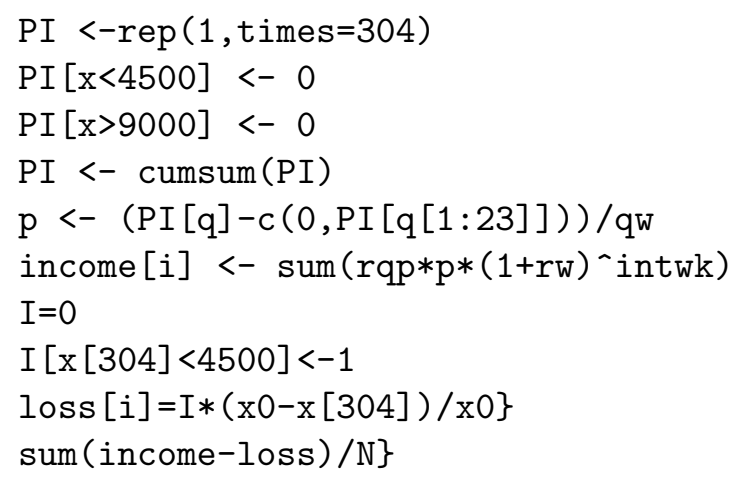

For example, using the parameters in (3.4), the step size $\Delta t=6 / 30400$ and the sample size 10000, by performing

$\operatorname{CIRP5}(6749.09,0.25,5200,10,0.02875,0.065,100,1000)$

we get the approximate MPR $=0.3320692$. This indicates that Plan P5 is much better than a 6-year fixed rate bound, if the Index follows the CIR model (3.2) with the parameters specified in (3.4). This is not surprising as the mean index is 5200 and the starting index level is 6749.09 and hence the index is very likely to be in the range from 4500 to 9000 in the next a few years.

\section{Conclusions}

In this paper, we have discussed five typical savings plans linked to the UK FTSE 100 Index. For some of them, we have obtained theoretical formulas for the MPRs which can either be computed explicitly or numerically. For most of them, we have established the approximation theory which enables us to perform Monte Carlo simulations to obtain the approximate MPRs. The theory established in this paper can be applied to other stock market linked savings accounts.

\section{Appendix}

The daily closing levels of FTSE 100 in year 2013:

[1] $6027.37 \quad 6047.34 \quad 6089.84 \quad 6064.58 \quad 6053.63 \quad 6098.65 \quad 6101.51 \quad 6121.58 \quad 6107.86$

[10] $6117.31 \quad 6103.98 \quad 6132.36 \quad 6154.41 \quad 6180.98 \quad 6179.17 \quad 6197.64 \quad 6264.916284 .45$

[19] $6294.416339 .196323 .11 \quad 6276.88 \quad 6347.24 \quad 6246.84 \quad 6282.76 \quad 6295.34 \quad 6228.42$

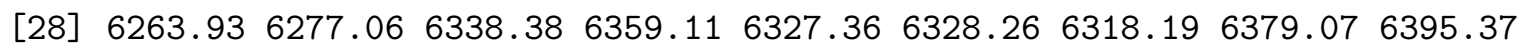

[37] $6291.54 \quad 6335.70 \quad 6355.37 \quad 6270.44 \quad 6325.88 \quad 6360.81 \quad 6378.60 \quad 6345.63 \quad 6431.95$

$\begin{array}{llllllllllll}{[46]} & 6427.64 & 6439.16 & 6483.58 & 6503.63 & 6510.62 & 6481.50 & 6529.41 & 6489.65 & 6457.92\end{array}$

[55] $6441.32 \quad 6432.70 \quad 6388.55 \quad 6392.76 \quad 6378.38 \quad 6399.37 \quad 6387.56 \quad 6411.74 \quad 6490.66$

[64] $6420.28 \quad 6344.12 \quad 6249.78 \quad 6276.94 \quad 6313.21 \quad 6387.37 \quad 6416.14 \quad 6384.396343 .60$

[73] $6304.58 \quad 6244.21 \quad 6243.67 \quad 6286.596280 .62 \quad 6406.12 \quad 6431.76 \quad 6442.596426 .42$

[82] $6458.02 \quad 6430.12 \quad 6451.296460 .71 \quad 6521.46 \quad 6557.30 \quad 6583.486592 .74 \quad 6624.98$

[91] $6631.76 \quad 6686.06 \quad 6693.55 \quad 6687.80 \quad 6723.06 \quad 6755.63 \quad 6803.87 \quad 6840.27 \quad 6696.79$

$\begin{array}{lllllllllllll}{[100]} & 6654.34 & 6762.01 & 6627.17 & 6656.99 & 6583.09 & 6525.12 & 6558.58 & 6419.31 & 6336.11\end{array}$

[109] $6411.996400 .45 \quad 6340.08 \quad 6299.45 \quad 6304.63 \quad 6308.26 \quad 6330.496374 .216348 .82$

$\left[\begin{array}{lllllllllllll}{[118]} & 6159.51 & 6116.17 & 6029.10 & 6101.91 & 6165.48 & 6243.40 & 6215.47 & 6307.78 & 6303.94\end{array}\right.$

$\left[\begin{array}{lllllllllllll}{[127]} & 6229.87 & 6421.67 & 6375.52 & 6450.07 & 6513.08 & 6504.96 & 6543.41 & 6544.94 & 6586.11\end{array}\right.$

$\left[\begin{array}{llllllllllll}{[136]} & 6556.35 & 6571.93 & 6634.36 & 6630.67 & 6623.17 & 6597.44 & 6620.43 & 6587.95 & 6554.79\end{array}\right.$

$\begin{array}{llllllllllllll}{[145]} & 6560.25 & 6570.95 & 6621.06 & 6681.98 & 6647.87 & 6619.58 & 6604.21 & 6511.21 & 6529.68\end{array}$

[154] $6583.396574 .34 \quad 6611.94 \quad 6587.43 \quad 6483.34 \quad 6499.996465 .73 \quad 6453.46 \quad 6390.84$ 
$\begin{array}{lllllllllll}{[163]} & 6446.87 & 6492.10 & 6440.97 & 6430.06 & 6483.05 & 6412.93 & 6506.19 & 6468.41 & 6474.74 \\ {[172]} & 6532.44 & 6547.33 & 6530.74 & 6583.99 & 6588.43 & 6588.98 & 6583.80 & 6622.86 & 6570.17 \\ {[181]} & 6558.82 & 6625.39 & 6596.43 & 6557.37 & 6571.46 & 6551.53 & 6565.59 & 6512.66 & 6462.22 \\ {[190]} & 6460.01 & 6437.50 & 6449.04 & 6453.88 & 6437.28 & 6365.83 & 6337.91 & 6430.49 & 6487.19 \\ {[199]} & 6507.65 & 6549.11 & 6571.59 & 6576.16 & 6622.58 & 6654.20 & 6695.66 & 6674.48 & 6713.18 \\ {[208]} & 6721.34 & 6725.82 & 6774.73 & 6777.70 & 6731.43 & 6734.74 & 6763.62 & 6746.84 & 6741.69 \\ {[217]} & 6697.22 & 6708.42 & 6728.37 & 6726.79 & 6630.00 & 6666.13 & 6693.44 & 6723.46 & 6698.01 \\ {[226]} & 6681.08 & 6681.33 & 6674.30 & 6694.62 & 6636.22 & 6649.47 & 6654.47 & 6650.57 & 6595.33 \\ {[235]} & 6532.43 & 6509.97 & 6498.33 & 6551.99 & 6559.48 & 6523.31 & 6507.72 & 6445.25 & 6439.96 \\ {[244]} & 6522.20 & 6486.19 & 6492.08 & 6584.70 & 6606.58 & 6678.61 & 6694.17 & 6750.87 & 6731.27 \\ {[253]} & 6749.09 & & & & & & & & \end{array}$

\section{Acknowledgements}

The authors would like to thank the referees for their helpful comments and suggestions. The authors would also like to thank the Leverhulme Trust (RF-2015-385), the EPSRC (EP/E009409/1), the Royal Society of London (IE131408), the Royal Society of Edinburgh (RKES115071), the London Mathematical Society (11219), the Edinburgh Mathematical Society (RKES130172), the National Natural Science Foundation of China $(71573043,71473039)$ and the Ministry of Education (MOE) of China for their financial support.

\section{References}

[1] Black, F. and Scholes, M., The pricing of options and corporate liabilities, J. Polit. Econ. 81 (1973), 637-659.

[2] Chan, K.C., Karolyi, G.A., Longstaff, F.A. and Sanders, A.B., An eMPRrical comparison of alternative models of the short-term interest rate, The Journal of Finance, 47(3) (1992), 1209-1227.

[3] Cox, J.C., Ingersoll, J. and Ross, S.A., A theory of the term structure of interest rates, Econometrica 53 (1985), 385-407.

[4] Elliott, R. and Kopp, E., Mathematics of Financial Markets, Springer, 1998.

[5] Heston, S.I., A closed-form solution for options with stochastic volatility with applications to bond and currency options, Rev. Financ. Stud. 6 (1993), 327-434.

[6] Higham, D.J. and Mao, X., Convergence of Monte Carlo simulations involving the meanreverting square root process, J. Comput. Financ. 8 (2005), 35-61.

[7] Hull, J. and White, A., The pricing of options on assets with stochastic volatilities, J. Financ. 42 (1987), 281-300.

[8] Hobson, D.G. and Rogers, L.C.G., Complete Models with Stochastic Volatility, Math. Financ. 8 (1998), 27-48.

[9] Kloeden, P.E. and Platen, E., Numerical Solution of Stochastic Differential Equations, Springer-Verlag, 1992.

[10] Lewis, A.L., Option Valuation under Stochastic Volatility : with Mathematica Code, Finance Press, California, 2000. 
[11] Mao, X., Stability of Stochastic Differential Equations with Respect to Semimartingales, Longman Scientific and Technical, 1991.

[12] Mao, X., Exponential Stability of Stochastic Differential Equations, Marcel Dekker, 1994.

[13] Mao, X., Stochastic Differential Equations and Applications, 2nd Edition, Horwood, 2007.

[14] Merton, R.C., The theory of rational optional pricing, Bell J. Econ. Manag. Sci. 4 (1973), $141-183$.

[15] Nowman, K.B., Gaussian estimation of single-factor continuous time models of the term structure of interest rate, The Journal of Finance 52(4) (1997), 1695-1706.

[16] Samuelson, P., Rational theory of warrant pricing, Industrial Management Review 6 (1965), $13-39$.

[17] Vasicek, O., An equilibrium characterisation of the term structure, Journal of Financial Economics 5 (1977), 177-188.

\section{Figures}

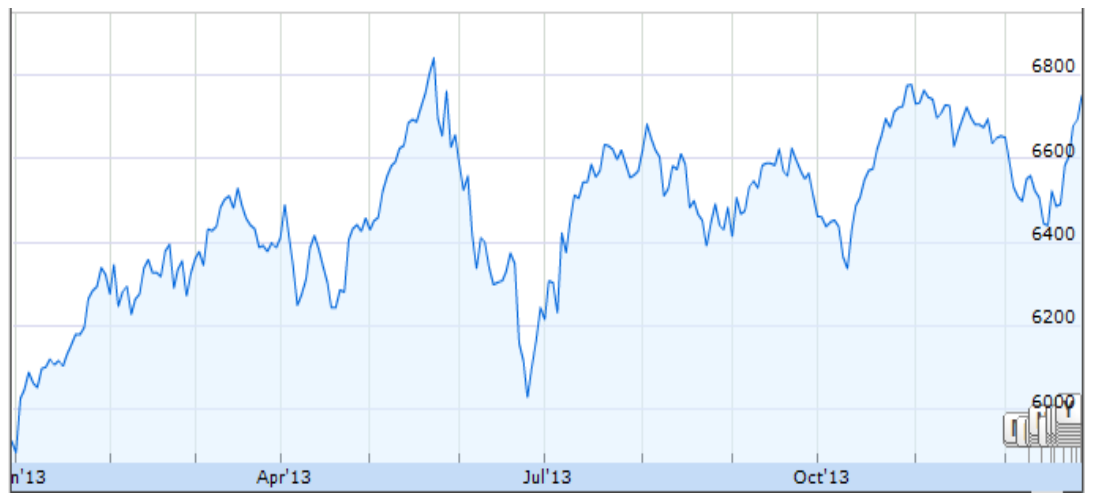

Figure 1: FTSE 100 Index: 2013

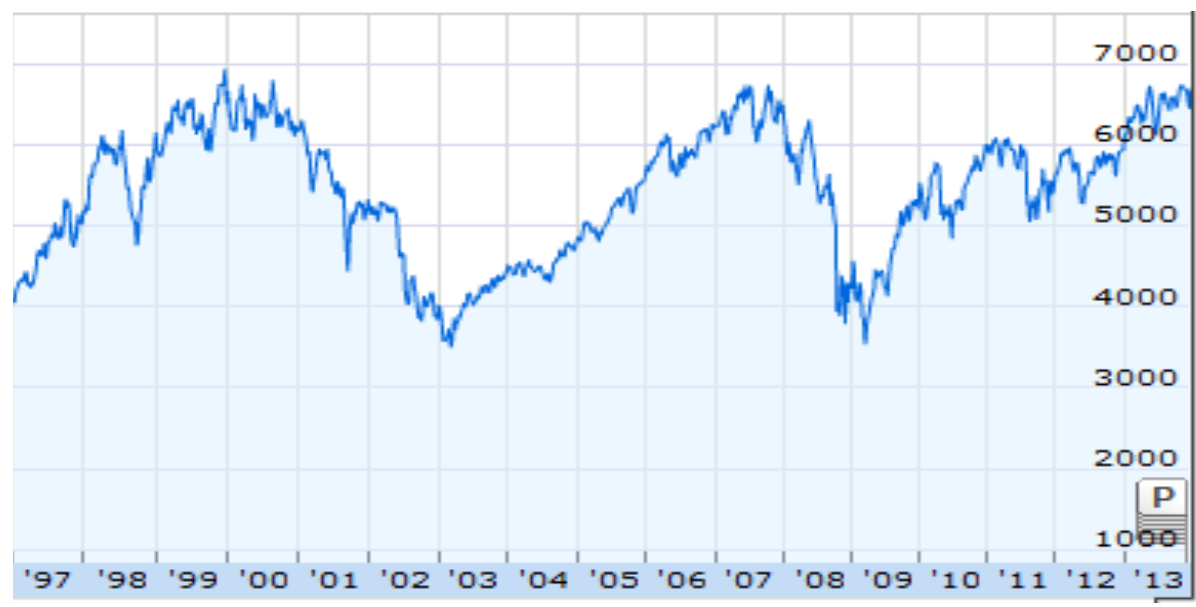

Figure 2: FTSE 100 Index: 1997-2013 


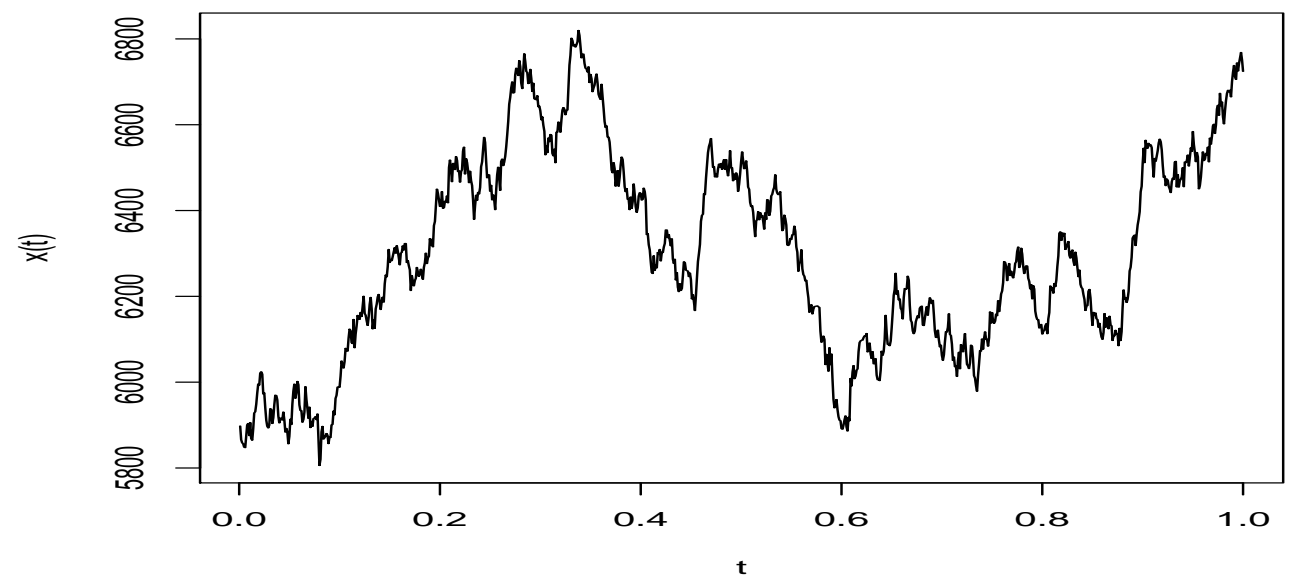

Figure 3: A sample path of the GBM

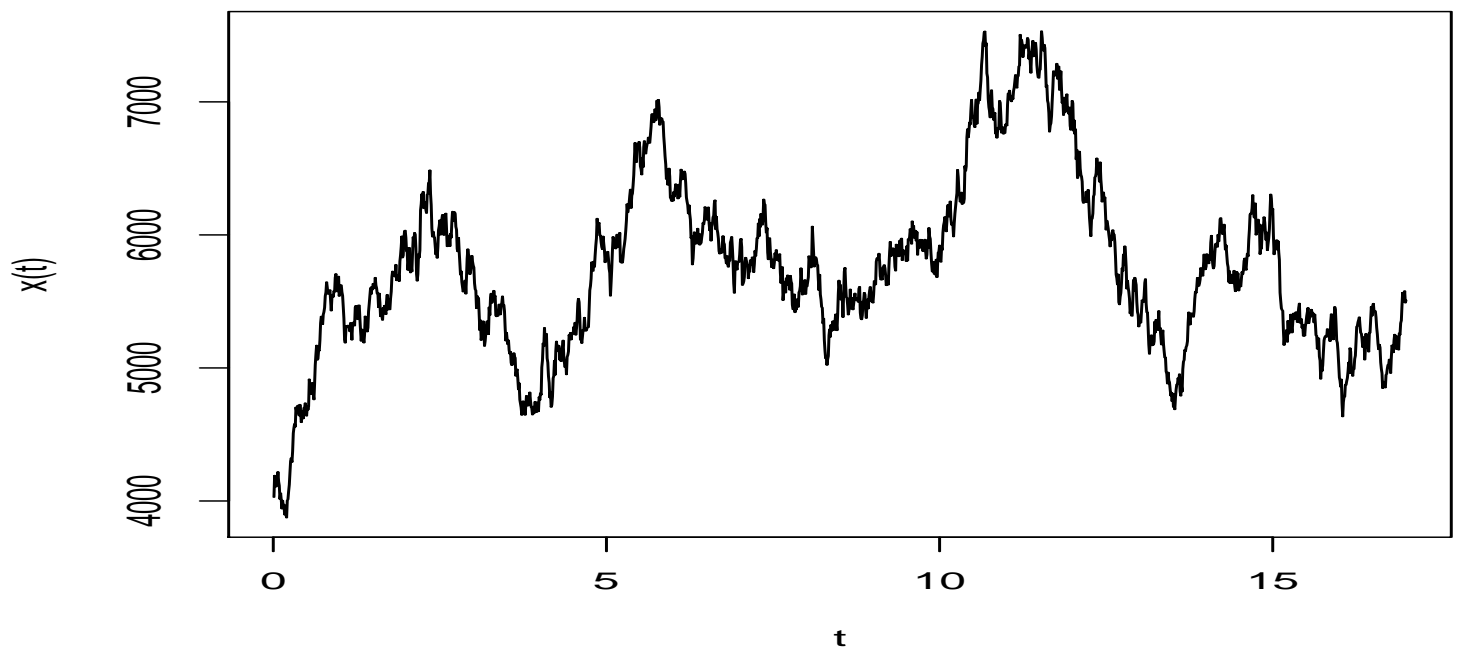

Figure 4: A sample path of the CIR model. 